Itinéraires Itinéraires

Littérature, textes, cultures

2009-2 | 2009

Caraïbe et océan Indien

\title{
La poétique du bateau dans la fiction mauricienne
}

\section{Namrata Poddar}

\section{OpenEdition}

\section{Journals}

Édition électronique

URL : http://journals.openedition.org/itineraires/276

DOI : 10.4000/itineraires.276

ISSN : 2427-920X

Éditeur

Pléiade

Édition imprimée

Date de publication : 1 juillet 2009

Pagination : 77-91

ISBN : 978-2-296-09639-4

ISSN : 2100-1340

Référence électronique

Namrata Poddar, «La poétique du bateau dans la fiction mauricienne », Itinéraires [En ligne], 2009-2 | 2009, mis en ligne le 03 septembre 2014, consulté le 01 mai 2019. URL : http:// journals.openedition.org/itineraires/276 ; DOI : 10.4000/itineraires.276

Ce document a été généré automatiquement le 1 mai 2019

\section{(c) (i) () $\Theta$}

Itinéraires est mis à disposition selon les termes de la licence Creative Commons Attribution - Pas d'Utilisation Commerciale - Pas de Modification 4.0 International. 


\title{
La poétique du bateau dans la fiction mauricienne
}

\author{
Namrata Poddar
}

1 Avant l'avènement des voyages aériens et de la cyber-technologie, c'était le bateau ${ }^{1}$ qui dominait l'imaginaire populaire comme médium par excellence du voyage et de la découverte de l'inconnu. La littérature occidentale en particulier, traversée qu'elle est par toute une histoire d'exploration, d'annexion et de colonisation, n'a cessé de revisiter le motif du bateau à diverses fins esthétiques. Dans cette optique, on pourrait parler d'une poétique du bateau qui met en scène des récits de voyages - glorieux ou périlleux : la découverte des terres inconnues, le commerce, la guerre, la colonisation, ou les transformations positives (chez un individu, voire une société entière), issues de la rencontre avec l'autre, souvent dans l'espace utopique d'une île lointaine².

2 La littérature exotique et moderne du XIX ${ }^{\mathrm{e}}$ siècle se situe dans cet héritage esthétique au sein duquel plusieurs artistes ont valorisé l'exil et l'errance, perpétuant un imaginaire des voyages et des vaisseaux marins comme catalyseurs de la découverte d'autrui, ainsi que des transformations conséquentes de soi, de la société et de l'art occidentaux ${ }^{3}$. Dans le riche répertoire des textes recelant un imaginaire idéalisé du bateau, c'est Georges (1843), le roman historique d'Alexandre Dumas, qui s'affirme, de la manière la plus évidente, comme un intertexte important et sur lequel nous reviendrons à plusieurs reprises. Le récit dumasien, dont l'action se déroule à l'île-de-France ${ }^{4}$, dévoile toute une gamme de héros marins (tels le colon, l'explorateur, le corsaire, le négrier, le capitaine), et il relate leurs exploits glorieux et spectaculaires à bord des vaisseaux qu'ils commandent avec brio. Tous sont des héros courageux dont les aventures ne font que renforcer l'aisance et la complicité avec les éléments naturels.

Dans la littérature et la pensée critique $\mathrm{du} \mathrm{xx}^{\mathrm{e}}$ siècle, et la théorie post-coloniale en particulier, le bateau consolide une poétique idéalisée, déjà répandue au sein des récits de guerre, de commerce, de colonisation, d'exil, culminant souvent en une certaine euphorie des liens établis entre les espaces, des transformations positives, fruits d'une esthétique pluraliste et postmoderne. Par exemple, Michel Foucault termine son article «Des espaces autres » en évoquant le bateau comme un espace hétérotopique par excellence, 
comme la plus grande réserve d'imagination, d'aventures et de rêves ${ }^{5}$. À la différence de cette vision idéalisée, Antonio Benitez-Rojo envisage le bateau dans ses diverses manifestations - la machine infernale, à l'origine des histoires de l'exploration, de la colonisation, de la révolution industrielle et du capitalisme contemporain (Benitez-Rojo, 1996 : 7-8). Paul Gilroy, souligne le rôle de connecteur du bateau et du Passage du Milieu (l'Atlantique) dans l'échange créateur des discours politico-culturels, et notamment dans la négociation des identités noires et (post)modernes (Gilroy, 1993: 4). Toujours en exposant la colonisation et ses conséquences ambivalentes, Édouard Glissant commence son essai Poétique de la Relation avec une image euphorique de la barque ouverte. Pour Glissant, le navire constitue à la fois le lieu d'un traumatisme originel pour le peuple antillais et le lieu d'un cri d'ordre poétique d'où peut surgir l'identité diasporique. Certes, il s'éloigne d'une poétique idéalisée du bateau pour dénoncer vivement la déportation des Africains vers le Nouveau Monde, leur angoisse face au déracinement et à l'inconnu, les maladies et la mort à bord (Glissant, 1989 : 17). Pourtant, à l'instar de Gilroy et de BenitezRojo, le navire chez Glissant est finalement allégorisé comme une matrice féconde, annonciatrice d'un (tout) monde créole - créateur, renouvelé et pluriel : « Nous pouvons dire maintenant que cette expérience du gouffre est la chose la mieux échangée [...] Nous crions le cri de poésie. Nos barques sont ouvertes, pour tous nous les naviguons » (Ibid.: 20-21).

4 Chez Glissant comme chez Gilroy ou Benitez-Rojo cependant, l'espace matriciel est déterminé par les trajectoires du bateau dans ce que Gilroy a appelé « l'Atlantique noir », c'est-à-dire l'espace situé entre les côtes de l'Atlantique, l'Europe et le Nouveau Monde. Cette étude entend élargir la problématique du bateau à un autre espace géographique et culturel, dont l'histoire est à la fois similaire et distincte de celle de l'Atlantique noir : l'océan Indien. Plus précisément, cette analyse examinera cinq romans issus de l'île Maurice : Le Voyage de Delcourt d'Alain Gordon-Gentil (2001), Ceux qu'on jette à la mer de Carl De Souza (2001), Le Silence des Chagos de Shenaz Patel (2005), Les Rochers de Poudre d'Or (2003) et Le Dernier Frère (2007) de Nathacha Appanah ${ }^{6}$. Centrée sur la problématique du bateau, elle tentera de répondre aux questions suivantes: comment la fiction mauricienne représente-t-elle le bateau? Dans quelle mesure les romans mauriciens déstabilisent-ils une poétique maritime, bien répandue et perpétuée par la littérature exotique et moderne (Dumas, Baudelaire, Rimbaud) ou la critique postmoderne (Foucault, Glissant, Gilroy, Benitez-Rojo) ? S'il se formule effectivement dans les textes mauriciens une autre poétique du bateau, quelle en est la spécificité?

\section{Nathacha Appanah : Les Rochers de Poudre d'Or}

5 Les Rochers de Poudre d'Or (2003) de Nathacha Appanah met en scène l'émigration des travailleurs engagés indiens, embauchés par des Anglais en Inde à la fin du xix siècle pour travailler dans les plantations sucrières à l'île Maurice, les deux pays étant des colonies britanniques à l'époque. Parmi les allusions éparses et brèves aux vaisseaux transportant les Indiens vers la terre promise de Maurice, c'est l'Atlas qui domine le texte d'Appanah. Après avoir quitté le port de Calcutta le 23 avril 1892 pour faire escale dans d'autres ports indiens, l'Atlas accueille des Indiens qui désirent échapper à la misère socio-économique de l'Inde coloniale, encore aggravée par le système des castes et les normes patriarcales. 
Divisé en deux parties symétriques et raconté majoritairement à la troisième personne, le récit d'Appanah souligne la perspective des émigrants indiens, les raisons diverses de leur départ et le sort sordide qui les attend sur les plantations sucrières. D'ailleurs, la partie centrale du texte (située vers la fin de la première partie) traitant le voyage en soi est un récit à la première personne, présenté par le biais du journal d'un colon anglais raciste, médecin à bord: le docteur Grant. Ce récit (à la première personne) dans le récit (à la troisième personne) permet de peindre le voyage infernal de l'intérieur, tout en rendant encore plus poignante l'expérience migratoire des travailleurs indiens. Le regard condescendant et xénophobe de Grant déshumanise les Indiens, confinés dans la cale. Le docteur Grant livre ce commentaire : «le sentiment de pitié avait complètement disparu et [...] je prenais plaisir à ce spectacle. Je les regardais, là, bêtes apeurées, et ça me donnait une furieuse envie de rire » (61). Le spectacle dont parle Grant est celui, navrant, d'un bateau perçu comme un espace surpeuplé, où s'entasse une masse d'Indiens « les uns sur les autres, en grappes » (78). L'Atlas, c'est d'ailleurs l'image d'un bateau en proie à l'océan monstrueux - «La pluie nous noyait et la mer ouvrait sa gueule béante pour avaler et recracher le bateau » (80) -, le rappel du désespoir à l'état pur - « La cale sentait le corps rance, la pisse, la crasse. J'ai pensé que si la misère devait avoir une odeur, ce serait cellelà » (78) - et la tyrannie d'une traversée qui refuse de se terminer7.

7 Le bateau dans Les Rochers de Poudre D'Or n'a ni le « rythme doux, et paresseux, et lent » du «Beau Navire » (Baudelaire, 1954 : 125) baudelairien, ni la promesse émancipatoire d'une Poétique de la Relation, fécondée par «la barque ouverte " (Glissant, 1990 : 17-21) chez Glissant. On est également bien loin ici de l'aventure maritime fort spectaculaire et épique qui clôt le roman historique de Dumas, Georges. Son dernier chapitre, intitulé « Le Combat ", dévoile un tête-à-tête féroce entre les deux anciens ennemis mortels, la frégate anglaise le Leycester et la Calypso, la corvette du corsaire français et du négrier Jacques (le frère aîné de Georges) qui en sort victorieux. Bien sûr, à la faveur de leur combat maritime, ces deux bateaux personnifient l'histoire de l'Empire français et sa victoire symbolique dans la fiction, à l'époque où la France venait de céder son ancienne colonie, l'île-de-France, à la Grande Bretagne.

En revanche, l'Atlas est un «bateau maudit» (57), dénué de toute grandeur, dont le spectacle quotidien témoigne de l'impuissance et de l'indifférence face à la mort volontaire ou involontaire - «Sur les bateaux, la mort guette et je n'y peux rien » (58) -, aux maladies $(72,83,85)$, aux hallucinations et à la folie qui frappent non seulement les émigrants indiens mais aussi le seul colon qui meurt à bord : le docteur Grant. Ce dernier, à chacune de ses traversées, ne lit qu'un seul livre, La Tempête de Shakespeare - «Sur un bateau, en direction des îles, en compagnie de fous, il n'y a rien de plus, comment dire, approprié » (58) - et il se suicide finalement, hanté par l'image d'un Prospero noyé qu'il confond soit avec William, l'autre colon à bord, soit avec les Indiens morts $(71,101,102)$. Aussi, l'Atlas est un texte palimpseste qui, tout en faisant écho au récit shakespearien, en permet une réécriture subversive. Le héros à bord du bateau n'est plus le sorcier européen, commandant aux éléments naturels, surtout à la mer. Le Prospero d'Appanah est plutôt une figure fantomatique et ambivalente, incarnant également la figure de l'exploiteur européen et des exploités indiens, tous également tyrannisés par la fureur océanique et le voyage infernal. 


\section{Alain Gordon-Gentil : Le Voyage de Delcourt, et Nathacha Appanah : Le Dernier Frère}

Dans Le Voyage de Delcourt d'Alain Gordon-Gentil, le bateau est de nouveau signe de la migration forcée, de la trahison, des maladies et de la mort provoquées par des trajets maritimes atrocement longs et difficiles. Cet ouvrage relate le voyage du protagoniste Delcourt, son désir de liberté et d'amour absolu, dénué de tout lien terrestre (national, communautaire ou familial) et sa rencontre amoureuse avec Marika Linderbaum, une réfugiée juive à Maurice. L'intrigue principale se noue avec l'histoire collective et l'expérience maritime d'un groupe de migrants juifs, pour lesquels la mer n'est guère une vocation, mais le seul espoir d'évasion vers une vie meilleure.

Dans le répertoire des vaisseaux marins, ceux qui dominent le récit de Gordon-Gentil sont le Johan de Witt, le Nieuw Zeeland et l'Hélios, des bateaux transportant des Juifs fuyant la persécution nazie en Allemagne et en Europe centrale pendant la Seconde Guerre mondiale. L'Hélios voguant sur les «eaux noires » (141) du Danube transporte mille six cents juifs européens vers la terre promise de Palestine, laquelle était à l'époque sous mandat britannique. À leur arrivée en Palestine où la situation politique est estimée trop précaire, les migrants juifs sont rapidement embarqués sur le Johan de Witt et le Nieuw Zeeland pour l'île Maurice, où ils sont détenus dans la prison de Beau Bassin de 1940 à 1945. En fait, les trois bateaux servant à la migration juive dans Le Voyage de Delcourt annoncent l'Atlantic, le seul vaisseau marin qu'évoque l'ouvrage le plus récent de Nathacha Appanah, Le Dernier Frère (2007). Semblable à la navigation du Johan de Witt, du Nieuw Zeeland et de l'Hélios, celle de l'Atlantic relie plusieurs espaces (des pays européens à l'océan Indien en passant par le Moyen-Orient), afin d'expulser des réfugiés juifs dans la prison de Beau Bassin à Maurice. De plus, comme dans l'ouvrage de Gordon-Gentil, Le Dernier Frère tisse le récit collectif de l'exode juif et de la rencontre historique entre Juifs et Mauriciens avec le récit plus personnel d'une tendre amitié entre les deux protagonistes : le petit Raj, fils d'un travailleur engagé indo-mauricien, et David, un des réfugiés juifs détenus à Maurice.

11 Ainsi, les bateaux transportant la communauté juive dans les deux ouvrages (Le Voyage de Delcourt et Le Dernier Frère) évoquent un espace inhospitalier, pareil à l'Atlas, qui expulse sa « cargaison humaine » (108) dans l'île-prison de Maurice. Donneuses de vie, ces arches de Noé sont les seuls moyens d'échapper à la persécution nazie dont les Juifs européens sont l'objet. Donneurs de mort, tous ces navires engendrent des maladies, la mort et une certaine désillusion concernant le voyage. Les eaux noires du Danube engloutissent le vieux père de Marika alors que les Juifs quittent l'Europe, tout comme le kala pani (les eaux noires en hindi) avale le plus vieux des émigrants indiens dans Les Rochers de Poudre d'Or. De plus, du fait de leurs nombreuses escales (en Europe, au Moyen-Orient, dans l'océan Indien) en route vers la destination désirée, mais toujours reportée, de la Palestine, y compris le séjour long et inattendu à Maurice, les bateaux transportant des réfugiés juifs sont des symboles équivoques du désir et du désir constamment différé.

À l'image de ces vaisseaux historiques, les passagers sont des signes de l'espoir et du désespoir. Si le plus vieil émigrant dans Les Rochers de Poudre d'Or rappelle à Grant le héros shakespearien, Prospero, dans la mesure où les deux sont « déterminé[s] et désespéré[s] » (62), les réfugiés juifs chez Gordon-Gentil sont « à la fois inquiets et soulagés. Soulagés de partir, mais inquiets du voyage qui s'annonçait difficile» (145). Il y a d'ailleurs «dans la 
détresse de ces gens une volonté inébranlable de traverser la vie avec dignité » (109). Ce mélange de sentiments opposés et cette condition ambiguë s'inscrivent ainsi dans la lignée de ces (anti)héros marins, tiraillés entre le désir et le refus de se confronter à la mer, entre la volonté de quitter leur terre et l'incapacité d'en (re)trouver une autre, hospitalière et sûre.

Si les bateaux dans les deux ouvrages d'Appanah se limitent à dévoiler l'histoire collective de deux peuples différents, des travailleurs indiens et des Juifs, les bateaux chez GordonGentil, tout en prenant à leur compte l'expérience collective du peuple juif, la transcendent. Ainsi, d'autres allusions aux vaisseaux mettent en relief les voyages de Delcourt: l'Explorateur Grand-Didier (19) marque un des premiers départs du héros à l'étranger en 1920, ses séjours et aventures variés ; suivi du Pierre-Loti qui le ramène vers Maurice, sa patrie, en 1938. Chez Delcourt, ces bateaux servent à souligner l'opposition binaire de la mer qui lui inspire une certaine liberté et de la terre qui emprisonne l'individu dans des liens et des devoirs sociaux. Ainsi que le suggèrent leurs noms, l'Explorateur Grand-Didier et le Pierre Loti font écho à un certain héritage littéraire, ivre d'exotisme, de la découverte d'autrui et de terres lointaines. Bien sûr, la volonté de s'exiler en quête de liberté et le désir du nomadisme (manifestes chez Delcourt) restent l'apanage d'un certain groupe social, souvent aisé et libre, offrant un contraste frappant avec l'expérience des migrants indiens et juifs qui sont marginalisés par leur situation historico-politique et existentielle. Il est alors peu surprenant que Delcourt soit le fils d'un colon français, le riche héritier de plantations sucrières, auquel sa situation socioéconomique offre le luxe de choisir le nomadisme et le voyage comme vocation.

Et pourtant, malgré cette dimension individuelle et fortement distincte de l'histoire collective de la migration juive, l'ouvrage de Gordon-Gentil se clôt sur la folie du héros engendrée par le départ de sa bien-aimée, Marika. Celle-ci, lassée par l'intensité de l'amour chez Delcourt, décide de le quitter et de partir avec ses compatriotes juifs sur le Franconia en 1945, en route vers la Palestine. Le départ du Franconia de Maurice marque la victoire de la terre - symbole classique des liens sociaux - du passé et de la mémoire collective sur la liberté individuelle, l'amour et le présent, idéaux auxquels ce hérosnomade se voue - "Cette terre qui le laissait si indifférent et qui n'arrêtait pas de rattraper son destin à lui. Elle n'arrêtait pas de le trahir. Jamais nomade ne fut tant insulté » (203).

\section{Shenaz Patel : Le Silence des Chagos}

De la période coloniale à l'époque de la décolonisation, en passant par la Seconde Guerre mondiale, le bateau dans la fiction mauricienne ne cesse d'explorer la face cachée de l'histoire, de récolter des anti-récits de voyage, soulignant des expériences collectives de marginalité, de désillusion, voire de trahison, de maladies et de mort. Ainsi le roman de Shenaz Patel, Le Silence des Chagos (2005), dénonce l'un des plus grands silences et l'une des tragédies marquantes de l'histoire contemporaine. Ce texte raconte la déportation des Chagossiens de Diego Garcia, une des îles de l'archipel des Chagos, qui appartenait auparavant à Maurice, mais qui aujourd'hui fait partie des BIOT (British Indian Ocean Territories). À la suite d'un pacte tacite et politico-économique entre les gouvernements britannique, américain et mauricien ${ }^{8}$, la Grande Bretagne a accordé l'indépendance à l'île Maurice en 1968 à la condition de garder en sa possession l'archipel des Chagos. À la suite d'une évacuation forcée et soudaine de tous les Chagossiens, la Grande Bretagne a loué 
l'île de Diego Garcia au gouvernement américain, qui l'utilise aujourd'hui comme une de ses plus grandes bases militaires.

Pour envisager la gamme des vaisseaux marins qui scande l'ouvrage de Patel, deux moments textuels s'imposent. Dans le premier épisode ${ }^{9}$, le narrateur retrace la carrière maritime du Nordvaer, un bateau d'origine norvégienne, en énumérant ses rôles variés aux différents moments de son usage professionnel de 1956 à 1968 (133-138). Ayant d'abord servi comme bateau de tourisme et navire cargo dans la mer du Nord, le Nordvaer, que les lignes ferroviaires ont rendu caduque, fut vendu au gouvernement seychellois. Utilisé afin de fournir du ravitaillement aux îles de l'océan Indien, le Nordvaer se réjouissait de redevenir essentiel et attendu quand, un beau jour, il fut forcé par les autorités britanniques de trahir les Chagossiens. Certes, ce récit marin soulignant les aventures variées du Nordvaer et ses émotions opposées (euphoriques et dysphoriques), suggère un parallélisme fort avec «Le Bateau Ivre» de Rimbaud (1871), jusque dans le désir d'immobilité et de naufrage volontaire. Tandis que l'itinéraire du bateau rimbaldien se termine sur une expérience personnelle de désespoir et de désillusion à l'égard de l'idéal du voyage, le Nordvaer souffre du poids historique de la déportation chagossienne et de son impuissance devant l'avarice des hommes qui continuent à utiliser des bateaux à des fins (néo)coloniales. Autrement dit, le récit du Nordvaer souligne sa culpabilité, son désir de se rouiller et de couler pour avoir trahi, malgré lui, les Chagossiens qui l'attendaient pour le ravitaillement périodique.

Ce moment textuel est unique car le narrateur de Shenaz Patel présente l'histoire maritime du commerce, du tourisme et de la (néo)colonisation à travers la perspective d'un bateau qui usurpe la voix narratrice pour terminer lui-même son récit tragique. Fait rare dans le monde romanesque : le Nordvaer, un bateau, prend la parole comme un sujet actif, féminin et maternel, qui s'avoue hanté par les cris des Chagossiens et surtout du bébé prénommé Désiré né dans son ventre au moment de leur traversée. Le Nordvaer, cet autre gouffre-matrice ${ }^{10}$, ne cesse de regretter l'exode forcé des Chagossiens, soumis à une traversée infernale, longue et difficile avant d'être finalement expulsés sur la terre inhospitalière de Maurice. Aussi, le récit du Nordvaer, le bateau-qui-parle, est un rappel de l'ironie amère qui a recréé une forme de colonialisme dans l'espace archipélique de l'île Maurice alors même que la décolonisation était en cours. Le bateau chez Patel est d'ailleurs un rappel de la vulnérabilité particulière des espaces insulaires, qui deviennent, au gré de l'histoire, lieux stratégiques - base militaire ou site d'essais nucléaires - mis à la disposition, souvent malgré eux, des grandes puissances. Une étude comparée avec la fiction et l'histoire d'autres espaces insulaires comme l'atoll de Bikini, la Baie de Guantánamo, ou les îles Falkland serait, de ce point de vue, sans doute instructive.

Dans l'autre scène de l'ouvrage de Patel, le bateau expose le quotidien insulaire à travers l'expérience de la communauté de héros que constituent les pêcheurs. Ici, Désiré, un des protagonistes chagossiens (né dans le ventre du Nordvaer ainsi que nous l'avons mentionné), se fait embaucher par une compagnie maritime dans la capitale de Maurice, Port-Louis. Immigrant-réfugié (émigrant d'un îlot à une île) dans un espace archipélique, Désiré le pêcheur est l'un de ces héros marins, doublement marginalisés et forcés de prendre le large pour gagner leur vie, "parce que, dans la cité où ils [vivent], les logements [sont] trop exigus, le bruit trop envahissant. L'argent trop inaccessible. L'horizon trop barré » (122). Ainsi, nourri de (méta-) récits de voyage et malgré son scepticisme envers la mer, Désiré embarque sur une pirogue, prétendant faire « une pêche miraculeuse» (121). Or, le héros de Patel n'éprouve que désillusion devant le 
sentiment de claustrophobie à bord et l'absence complète de grandeur de son aventure maritime - «Il n'avait rêvé la mer qu'en images de grandeur, d'espace, de lumière. Il se retrouvait confiné dans un lieu au plafond bas, mal éclairé, où deux hommes ne pouvaient se croiser » (120) -, en contraste sordide avec les expériences des navigateurs américains et britanniques dont les « récits extatiques vantaient [au port] la vie de Robinson qu'ils ont menée » (116).

Avec Désiré à bord de son bateau-pêcheur, Le Silence des Chagos déconstruit davantage la représentation romantique de la mer et du bateau comme des espaces ouverts, inspirant un certain sentiment de mystère et de sublime. En effet, le récit ne cesse de souligner le malaise chez Désiré, complètement en proie à un bateau qu'il perçoit comme un objet purement mécanique, menaçant et étouffant :

Il la [la mer] sent, il pressent son manège, elle le liquéfie de l'intérieur, sournoise, elle a déminéralisé ses côtes et sa colonne vertébrale, dissous son estomac et ses viscères, il n'y a plus que son eau, là, qui veut sortir de l'outre de sa peau. Il a juste le temps de projeter son torse hors de la couchette. Sur le plancher de la cabine, il vomit à longs traits fiévreux. (127)

Avec le mal de mer de ce marin anti-héros, sa claustrophobie, sa réaction viscérale et son aliénation vis-à-vis du bateau et de la mer dans une description poignante de huit pages (120-127), la fiction mauricienne atteint l'apogée d'une poétique dysphorique du bateau.

\section{Carl De Souza : Ceux qu'on jette à la mer}

Ce que les textes d'Appanah, de Gordon-Gentil et de Patel démontrent en filigrane, De Souza le dénonce plus directement dans un récit de deux cents pages, dont l'action principale se déroule sur un bateau. Son ouvrage intitulé Ceux qu'on jette à la mer raconte ainsi l'expérience migratoire de boat-people chinois embarqués à bord du Ming Sing 23 et désireux d'échapper à leur patrie pour travailler dans l'espace démocratique de l'Amérique, et d'Haïti en particulier. Malgré des allusions brèves à divers vaisseaux dans le texte, le Ming Sing 23 en est sans doute le personnage principal. Quittant le port chinois de Kwan Chou, il prend à son bord des maçons, des électriciens, des étudiants en mécanique et des professeurs au chômage (19) pour un voyage long et douloureux, «plein de fantômes et de sang 》 (200), à travers la mer de Chine, ne demandant à ses passagers que « deux-petits-mois-de-patience » (102). Faisant tout d'abord escale en Malaisie (94), puis sur une île indiaocéanique (qui n'est pas sans évoquer Maurice), les passagers du Ming Sing 23 sont finalement rapatriés en Chine, après avoir réalisé qu'ils sont les victimes d'une escroquerie dont le principal responsable est le capitaine (193).

L'absence de repères spatio-temporels fixes chez De Souza signale la dimension universelle de cet anti-récit de voyage. L'expérience migratoire douloureuse des boatpeople chinois est analogue à celle des groupes marginalisés (par leur situation sociohistorique) que sont les travailleurs indiens, les réfugiés juifs et chagossiens ou les pêcheurs dont le quotidien est pénible. Raconté selon la perspective de l'un des passagers chinois, Tian Sen, ce récit à la première personne (alternant le je et le nous), ne cesse de souligner l'expérience collective d'anonymat et de marginalité extrême des passagers (51, $79,80,115,192,197)$. Comme le met en évidence le titre du récit, Ceux qu'on jette à la mer, ces antihéros marins sont doublement marginalisés car ils sont des Chinois pauvres et des émigrants clandestins soucieux d'échapper au régime communiste de la Chine. À l'image des anti-héros présents à bord des vaisseaux que nous avons précédemment envisagés, le 
Ming Sing 23 transportant des boat-people souligne autant le calvaire des parias sociaux que des réfugiés en général, pour qui la terre et la mer restent des espaces aussi inhospitaliers : « Nous sommes d'irrémédiables déracinés, d'éternels réfugiés, la mer nous refoule, la terre ne sait nous retenir, nous sommes pris dans un ressac incessant, violent et silencieux, qui n'en finit pas de voler nos rêves » (203-204).

Dans une large mesure, les caractéristiques du Ming Sing 23 renvoient à celles d'autres bateaux de la fiction mauricienne que nous avons énumérées : le bateau comme espace claustrophobe débordant de passagers, où «jamais la présence des autres n'était aussi oppressante » (12); comme lieu envahi par la peur (18), l'odeur nauséabonde, la crasse $(46,47)$, les maladies, la mort, les viols $(47,128,130)$, les fantômes $(65,68)$ et la folie latente du narrateur-protagoniste Tian Sen ${ }^{11}$. Plus loin, le narrateur éprouve un dégoût et une désillusion similaires envers le voyage, les vaisseaux et les aventures maritimes : « Le Ming Sing 23, souffleté par des eaux traîtresses sonnait creux ", ou encore, "Le Ming Sing 23, c'est la statue de la déesse bafouée et estropiée » (109). Entre les " eaux traitresses » (De Souza), le «kala pani» (Appanah) et les « eaux noires» (Gordon-Gentil), la fiction mauricienne ne se départ guère d'un imaginaire monstrueux de la mer et des bateaux.

Si le bateau est généralement envisagé comme emblématique du mouvement, dans l'œuvre de De Souza, il signale sans cesse la lenteur, l'attente et l'inertie. Le voyage à bord du Ming Sing 23 traduit l'impossibilité de quitter la terre d'origine, la mer de Chine, et d'atteindre la destination désirée. C'est le drame d'un voyage qui refuse de finir, voire de simplement se poursuivre, c'est la tyrannie sans fin d'être cloué et perdu dans le passage du milieu, ce dernier n'étant plus l'Atlantique, mais la mer de Chine et l'océan Indien. L'anti-voyage à bord du Ming Sing 23 dépouille ainsi ses passagers de toute notion du temps, les figeant dans un présent éternel et oppressant : «La façon dont on dispose de notre temps depuis le départ est pour moi la chose la plus dégradante que j'aie subie depuis longtemps. Attendre, toujours attendre, les ordres, la nourriture. Attendre le malheur » (80). Dans cette traversée, aucun sentiment de sublime devant la grandeur de l'océan et la nature; aucun «Bateau ivre » à la Rimbaud ni même aucune "Mer » à la Trenet dont la chanson populaire prétend «bercer le cœur» de son auditoire avec ses belles métaphores marines. Le Ming Sing 23 incarne la misère d'une traversée réduite à la tyrannie du présent éternel, à l'attente, à l'inertie et aux besoins corporels :

Nous ne sommes pas partis, nous n'avons pas pris le large, nous sommes dans un entonnoir bleu, la mer et le ciel se sont enroulés en cornet autour de nous, il n'y a plus d'horizon, pas de vrai ciel [...] alors, je redescends et vais faire comme les autres. Jouer aux cartes, jurer, manger, chier, me branler, vomir... (44)

Plus encore que Le Silence des Chagos, le récit de De Souza est marqué par la figure du bateau : objet purement matériel et métallique. Ainsi, le Ming Sing 23 est caractérisé par un lexique de la mécanique : « les vibrations spasmodiques du moteur » (60), « le paisible ronronnement " (76), «la ferraille» (159), la référence aux soucis incessants d'approvisionnement, au gasoil, au carburant ou aux rations en baisse $(155,156,160,161$, 176).

Enfin, laissons le dernier mot au narrateur-protagoniste Tian Sen dont la confession à bord du Ming Sing 23 résume la poétique de De Souza et, au-delà, la poétique mauricienne du bateau. Son aveu de non-représentation, d'échec du langage pour représenter l'aventure ratée, l'agonie et le calvaire maritimes des réfugiés s'impose comme une réponse virulente à un héritage littéraire marqué par le désir intarissable du voyage et de la découverte d'autrui : 
J'ai honte de ce voyage, honte d'en faire partie [...]. La seule idée de me trouver ici m'écœure. Je ressasse ce voyage de misère en attendant la vague salutaire [...]. Je voudrais vous écrire, quoi, je ne sais pas, quelque chose d'important de ces quelques jours ou sur ce que j'ai vécu avant [...]. Mais impossible de me lever pour me chercher le plastique, figé par le sommeil et par le mal de mer [...]. Je me réveille en sursaut, de temps à autre, pour vous dire quelque chose d'important qui m'échappe immédiatement. (47-48)

\section{Une poétique dysphorique de la souffrance et de l'exil}

Nous avons examiné les représentations du bateau dans cinq romans mauriciens, ce qui nous a permis d'analyser l'aventure maritime de divers groupes inscrits dans différentes périodes de l'histoire : les travailleurs indiens à la fin du XIX ${ }^{e}$ siècle (Les Rochers de Poudre d'Or), les réfugiés juifs pendant la Seconde Guerre mondiale (Le Voyage de Delcourt et Le Dernier Frère), les réfugiés chagossiens au moment de l'indépendance mauricienne, les pêcheurs (Le Silence des Chagos) et les boat-people chinois (Ceux qu'on jette à la mer). En insistant sur l'exil douloureux de parias sociaux issus de différents espaces, le bateau offre une réponse cinglante à tout un répertoire littéraire et populaire de héros marins : capitaine, explorateur, colon, corsaire, touriste, voire poète. L'exemple le plus pertinent en serait Jacques, l'un des héros dumasiens de Georges. Ayant « une vocation décidée pour la mer » (Dumas, 1974 : 219) dès son enfance, Jacques se fait marin et devient ensuite capitaine. Il poursuit une carrière, fort réussie, de négrier, commerçant et corsaire français. En fait, rien n'est plus éloigné de la poétique mauricienne du bateau que ce portrait du héros dumasien, inséparable de sa corvette la Calypso et en communion totale avec les éléments naturels :

Jacques était éternellement l'homme de la circonstance, universel en ce qui concernait l'art nautique; sachant, dans une bataille ou dans une tempête, commander la manœuvre comme le premier amiral venu, et faisant, dans l'occasion, un nœud à la marinière aussi bien que le dernier mousse. Avec Jacques, jamais de repos, et, par conséquent, jamais d'ennui. Chaque jour amenait une amélioration dans l'arrimage et dans le gréement de la goélette. Jacques aimait la Calypso comme on aime une maîtresse; aussi était-il éternellement préoccupé d'ajouter quelque chose à sa toilette. (230)

$\mathrm{Au}$ contraire, chez les antihéros de la fiction mauricienne, le voyage en mer n'est guère une vocation, mais une situation forcée, imposée par la misère ou la situation sociopolitique. À rencontre d'une tradition littéraire (romantique, exotique, moderne et postmoderne) qui a perpétué l'image du bateau comme un espace ouvert, donnant sur l'infini de la mer et le séjour insulaire comme catalyseur des transformations positives, le roman mauricien contemporain représente le bateau et l'île comme des espaces étouffants qui ne permettent aucune transformation chez ses héros. Signe ambivalent de vie et de mort, d'espoir et de désespoir, le bateau n'inspire qu'une désillusion aiguë, engendrée par une destination à jamais différée - que ce soit l'eldorado imaginé de Maurice chez les travailleurs indiens, la terre promise de Palestine chez les migrants juifs, le paradis perdu du pays natal chez les réfugiés chagossiens, l'espace démocratique d'Amérique chez les boat-people chinois ou tout simplement la possibilité de gagner sa vie chez les pêcheurs.

Dans une certaine mesure, la poétique mauricienne du bateau renvoie à la vogue récente de la critique postcoloniale qui s'est approprié l'image du bateau pour dénoncer les douleurs de la migration forcée et le drame colonial. Et pourtant, l'imaginaire mauricien 
ne dépasse pas la matérialité du voyage pour métaphoriser le bateau comme catalyseur et annonciateur d'une esthétique plurielle, hybride et postmoderne. Il développe, au contraire, une obsession pathologique du bateau, lequel est perçu comme un espace clos, inhospitalier, purement mécanique, et souligne sans cesse les maux qui frappent ses passagers.

\section{L'ouverture d'un espace transculturel}

Il serait toutefois important de noter qu'une poétique dysphorique du bateau n'est pas le fruit unique de l'imaginaire mauricien. Par exemple, une semblable poétique, marquée par la chaleur inhumaine, la monotonie, l'instinct de mort à bord, se dégage de l'aventure maritime des boat-people haïtiens échouant sur une plage de Floride qu'évoque Passages (1991) de l'écrivain haïtien Émile Ollivier, ou de celle des Italiens émigrant en Amérique dans le récent film d'Emmanuelle Crialese, Nuovo Mondo (2006). Dans le même temps, la fiction mauricienne, outrepassant une vision dysphorique du bateau, recèle parfois des moments où la mer et le bateau deviennent, à l'image d'une poétique déjà établie, des espaces idéalisés. Par exemple, dans Pagli d'Ananda Devi (2001), l'héroïne folle Daya, accusée d'amour adultère avec Zil le pêcheur, est enfermée dans un poulailler par les villageois patriarcaux de Terre Rouge. D'une part, le thonier de Zil représente la vie difficile des pêcheurs : "Tout pêcheur porte en lui sa noyade » (58); «Sur le thonier, les jours sont longs. La rouille ronge la coque, poursuit les hommes jusque dans les entrailles du bateau et de leurs songes [...]. Le bateau sent la mort » (61). Mais, d'autre part, il évoque l'océan, la liberté, l'amour et un espace utopique dont rêve Daya pour échapper à la terre qu'elle perçoit comme un espace dystopique, carcéral et meurtrier. Cette perception binaire des éléments naturels domine déjà le récit dumasien Georges et revient chez Gordon-Gentil avec Le Voyage de Delcourt.

De ce fait, la spécificité de l'imaginaire mauricien se lit moins dans la présence unique d'une poétique dysphorique du bateau que dans sa récurrence systématique et dans la fonction que cette poétique maritime joue sur le plan esthétique et socio-historique. Nous avons signalé à plusieurs reprises l'existence d'un héritage littéraire européen qui a représenté l'île Maurice et son histoire à l'image de Soi - c'est-à-dire comme une île édénique constituant un antidote à l'ennui et à la modernité européenne, ou comme un trophée chéri des empires coloniaux. Or, la poétique mauricienne du bateau se détourne d'un regard eurocentriste, voire impérial, porté sur le voyage maritime, sur l'île et sur son histoire. Elle insiste sur la perspective des passagers de troisième classe pour lesquels l'île Maurice demeure un lieu de passage ou une destination finale. En s'offrant comme un réceptacle et comme le porte-parole de diverses communautés marginales, le bateau explore et dénonce les silences variés d'une histoire, ou le quotidien et la vie difficile des pêcheurs. Ensuite, tout en la dénonçant, il dépasse l'histoire européenne de l'impérialisme pour désigner d'autres centres de pouvoir et d'oppression comme le système des castes et des normes patriarcales de l'Inde, la persécution nazie, le néocolonialisme américain, le communisme chinois, etc.

Sur le plan esthétique, le bateau dans le roman mauricien retrace un réseau transculturel semblable. Tout en faisant écho aux textes canoniques de la littérature occidentale qu'il n'a de cesse de subvertir, il permet de mettre en relief ce qui est souvent vu comme les marges de la " grande littérature » mondiale. Grâce à la présence de chansons indiennes et juives et de récits chinois folkloriques à bord de l'Atlas (60), de l'Hélios (145) et du Ming 
Sing $23(54,55)$ respectivement, le bateau souligne le rôle suprême de la tradition orale, du folklore et de la performance pour alléger les difficultés de la vie chez les antihéros marins.

En résistant à un discours eurocentriste pour y révéler les marges ou en reliant une marge à l'autre pour en exposer des centres, la fiction mauricienne, grâce au bateau, relie, sur le plan historico-culturel, l'île Maurice aux différentes parties du monde: l'Europe, l'Inde, les États-Unis, le Moyen-Orient, la Chine et les archipels indiaocéaniques. Elle dépasse ainsi les structures dichotomiques de pouvoir, de temporalité et de géopolitique (colon/colonisé, Nord/Sud, Europe/Afrique, colonial/postcolonial, centre/ marge) qui informent souvent la littérature et la critique francophones postcoloniales. Autrement dit, c'est par une poétique de la marginalité, de l'insularité, de la claustrophobie et de l'inertie que le bateau, dans le roman mauricien, ouvre un espace transculturel où l'île Maurice est reliée au monde entier pour établir un véritable échange historico-esthétique. C'est peut-être dans cette fonction paradoxale qu'il faudrait voir la singularité de la poétique mauricienne du bateau.

\section{BIBLIOGRAPHIE}

Appanah, Nathacha, 2003, Les Rochers de Poudre d'Or, Paris, Gallimard, coll. « Continents Noirs ». -, 2007, Le Dernier Frère, Paris, Éditions de l'Olivier.

Baudelaire, Charles, 1954, Euvres, texte établi et annoté par Y.-G. Le Dantec, Paris, Gallimard, coll. «Bibliothèque de la Pléiade».

Benitez-Rojo, Antonio, 1996 [1992], The Repeating Island, Durham, Duke University Press.

De Souza, Carl, 2001, Ceux qu'on jette à la mer, Paris, Éditions de l'Olivier.

Devi, Ananda, 2001, Pagli, Paris, Gallimard.

Dumas, Alexandre, 1974 [1843], Georges, éd. présentée, établie et annotée par Léon-François Hoffmann, Paris, Gallimard.

Foucault, Michel, 1984, « Des espaces autres » [Conférence au Cercle d'Études Architecturales, 14 mars 1967], Architecture, Mouvement, Continuité, nº 5, p. 46-49.

Gilroy, Paul, 1993, The Black Atlantic: Modernity and Double Consciousness, Cambridge, Massachusetts, Harvard University Press.

Glissant, Édouard, 1990, Poétique de la Relation, Paris, Gallimard. Gordon-Gentil, Alain, 2001, Le Voyage de Delcourt, Paris, Julliard. Patel, Shenaz, 2005, Le Silence des Chagos, Paris, Éditions de l'Olivier.

Rimbaud, Arthur, 1972, Euvres Complètes, éd. établie, présentée et annotée par Antoine Adam, Paris, Gallimard, coll. « Bibliothèque de la Pléiade ». 


\section{NOTES}

1. Notre étude emploiera «bateau " comme un terme générique et synonyme de vaisseaux marins, tels le navire, la pirogue, la frégate, étant donné que c'est le terme «bateau » qui revient le plus souvent dans notre corpus mauricien.

2. Quelques exemples du canon occidental qui recèlent cette poétique maritime, seraient: L'Odyssée d'Homère, La Tempête de Shakespeare, Paul et Virginie de Bernadin de Saint-Pierre, La Vie et les aventures étranges et surprenantes de Robinson Crusoé de York de Daniel Defoe, Vendredi ou Les Limbes du Pacifique de Michel Tournier, Voyage à Rodrigues de J.M.G. Le Clézio.

3. Voir, à ce sujet, Jills Frank, Islands and the Modernists: The Allure of Isolation in Art, Literature and Science, North Carolina, McFarland and Company, Inc, 2006. Selon Frank, le «modernisme » en littérature s'inscrit dans la période 1890-1930. Il est marqué par un certain scepticisme envers les institutions sociales telles que la nation, l'empire, le mariage, l'Église, voire les distinctions de classe, de genre et de race. Dans son étude, Frank insiste sur les diverses façons dont les artistes modernes se sont approprié l'image d'une île lointaine et isolée afin d'imaginer et de représenter des modèles alternatifs de la société et de l'art.

4. C'est l'ancien nom de l'île Maurice qui était une colonie française pendant le XVIII ${ }^{\mathrm{e}}$ siècle, avant sa prise de possession et sa colonisation par la Grande Bretagne, du début du XIX siècle jusqu'à l'indépendance de l'île en 1968.

5. «Le bateau, c'est un morceau flottant d'espace, un lieu sans lieu, qui vit par lui-même, qui est fermé sur soi et qui est livré en même temps à l'infini de la mer et qui, de port en port, de bordée en bordée, de maison close en maison close, va jusqu'aux colonies chercher ce qu'elles recèlent de plus précieux en leurs jardins, vous comprenez pourquoi le bateau a été pour notre civilisation, depuis le $\mathrm{xvI}^{\mathrm{e}}$ siècle jusqu'à nos jours, à la fois non seulement, bien sûr, le plus grand instrument de développement économique [...], mais la plus grande réserve d'imagination. Le navire, c'est l'hétérotopie par excellence. Dans les civilisations sans bateaux les rêves se tarissent, l'espionnage y remplace l'aventure, et la police, les corsaires » (Foucault, 1984:49).

6. Notre étude ne suit pas l'ordre chronologique de publication des œuvres, elle se fonde sur l'analyse de la complexité des discours maritimes.

7. Le texte prévoit une période de six semaines pour un voyage aller simple de l'Inde à l'île Maurice.

8. Voir, à ce sujet, Jocelyn Chan Low, "Les Chagos: de Oil Islands à British Indian Ocean Territory ", Journal of Mauritian Studies, vol.3, $\mathrm{n}^{\circ}$ 1, Institut Mahatma Gandhi, Maurice, 2006, p. 50-69.

9. Notons que l'auteur personnifie ici le navire en lui conférant un statut de personnage et, à un moment donné, en lui donnant la parole.

10. Afin de représenter l'expérience de l'exil douloureux et la traversée difficile, Glissant utilise l'image de la barque qui transporte des esclaves africains vers le Nouveau Monde comme celle d'un gouffre-matrice : «Le ventre de cette barque-ci te dissout, te précipite dans un non-monde où tu cries. Cette barque est une matrice, le gouffre-matrice [...]. Car si tu es seul dans cette souffrance, tu partages l'inconnu avec quelques-uns, que tu ne connais pas encore. Cette barque est ta matrice, un moule, qui t'expulse pourtant. Enceinte d'autant de morts que de vivants en sursis» (Glissant, $1990: 18$ ).

11. Notons que la folie de Tian Sen n'est pas sans évoquer celle du docteur Grant dans Les Rochers de Poudre d'Or de Nathacha Appanah. 


\section{RÉSUMÉS}

Depuis l'Antiquité, les bateaux ont été investis d'un imaginaire littéraire en tant que médium du voyage et de la découverte de l'inconnu, phénomène particulièrement visible dans la tradition littéraire occidentale (exotique, moderniste et post-moderniste). On peut alors parler d'une poétique du bateau qui fait émerger le récit de voyages maritimes glorieux et périlleux, de découvertes géographiques, de commerces, de guerres, de colonisations, ou de transformations positives et de processus pluralistes. Cet article examinera les diverses représentations du bateau dans cinq romans mauriciens contemporains ainsi que les manières dont elles déstabilisent une poétique prédominante de célébration du bateau afin de finalement suggérer la singularité de la poétique mauricienne du bateau.

Since antiquity, boats have been invested with a literary imaginary as the medium for travel and the discovery of the unknown-a phenomena particularly conspicuous in the Western (exoticist, modernist and post-modemist) literary tradition. One may then speak of a poetics of the boat that foregrounds the narrative of glorious and perilous sea-voyages, geographical discoveries, trade, war, colonization; or of positive transformations and pluralist processes. This article will examine the various representations of the boat in five contemporary Mauritian novels, the ways in which they destabilize a prevalent, celebratory poetics of the boat in orderfinally to suggest the singularity of the Mauritian poetics of the boat.

\section{INDEX}

Mots-clés : insularité, voyage maritime, marginalité, exil, impérialisme

Keywords : insularity, sea-travel, marginality, exile, imperialism

\section{AUTEUR}

\section{NAMRATA PODDAR}

Université de Pennsylvanie 